\title{
Three-Dimensional Fourth-Order Time-Fractional Parabolic Partial Differential Equations and Their Analytical Solution
}

\author{
Yesuf Obsie Mussa (iD, Ademe Kebede Gizaw ${ }^{(D)}$, and Ayana Deressa Negassa ${ }^{(D}$ \\ Department of Mathematics, College of Natural Sciences, Jimma University, Jimma, Ethiopia \\ Correspondence should be addressed to Ademe Kebede Gizaw; kebedeademe2020@gmail.com
}

Received 1 August 2020; Revised 17 December 2020; Accepted 9 January 2021; Published 21 January 2021

Academic Editor: Chuangxia Huang

Copyright (C) 2021 Yesuf Obsie Mussa et al. This is an open access article distributed under the Creative Commons Attribution License, which permits unrestricted use, distribution, and reproduction in any medium, provided the original work is properly cited.

\begin{abstract}
In this study, the fractional reduced differential transform method (FRDTM) is employed to solve three-dimensional fourth-order time-fractional parabolic partial differential equations with variable coefficients. The fractional derivative used in this study is in the Caputo sense. A few important lemmas which are essential to solve the problems using the proposed method are proved. The novelty of this method is that it uses appropriate initial conditions and finds the solution to the problems without any discretization, linearization, perturbation, or any restrictive assumptions. Two numerical examples are considered in order to validate the efficiency and reliability of the method. Furthermore, the FRDTM solution when $\alpha=1$ is compared with other analytical methods available in the existing literature. Computational results are shown in tables and graphs. The obtained results revealed that the method is capable and simple to solve fractional partial differential equations. The software used for the calculations in this study is Mathematica 7 .
\end{abstract}

\section{Introduction}

In the last decade, fractional calculus has become an important mathematical tool in several branches of sciences and engineering due to its enormous numbers of applications ([1] and the references therein). What specifics fractional calculus is the existence of various approaches to define differentiation with the nonintegral order [2]. As a result, several definitions for fractional derivatives appear in the literature to present more accurate models for real life phenomena. Some of known fractional derivatives are Riemann-Liouville, modified Riemann-Liouville, Caputo, Hadmard, Erdélyi-Kober, Riesz, Grunwald-Letnikov, Marchaud, and others ([3] and the references therein). All known fractional derivatives satisfy one of the well-known properties of classical derivative, namely, the linear property. However, the other properties of classical derivative, such as the derivative of a constant is zero; the product rule, quotient rule, and the chain rule either do not hold or are too complicated for many fractional derivatives.

In recent years, fractional differential equations have received considerable attention owing to their applicability in different fields of sciences such as chemistry, biology, diffusion, control theory, rheology, viscoelasticity, and so on [4]. Consequently, the solution of FPDEs represents nowadays a vigorous research area for scientists, and finding approximate and exact solutions to FPDEs is an important task [5]. However, PDEs are commonly hard to tackle, and their fractional order types are more complicated [2,6-10]. Therefore, several methods such as the homotopy perturbation method [11], subODE method [12, 13], generalized tanh method [14], residual power series method $[14,15]$, and so on [16-21] are developed to obtain solutions of some nonlinear fractional differential equations. Most of these methods sometimes require complex and huge calculation in order to obtain approximate solutions. To overcome such difficulties and drawbacks, an alternative method, the so called the fractional reduced differential transform method (FRDTM), has been developed by Keskin and Oturanc [22]. FRDTM plays a vital role among all the listed methods because it takes small size computation, easy to implement as compared to other techniques [23]. Using this method, it is possible to find both exact and approximate solutions in a rapidly convergent power series form. FRDTM is a very reliable, efficient, and effective powerful computational technique for solving physical problems [24-26]. 
In the field of modern science and engineering, the fourth-order initial/boundary value problems are of great importance. For example, airplane wings, bridge slabs, floor systems, and window glasses are being modeled as plates subjected to different types of end supports which are successfully described in terms of fourth-order PDEs $[27,28]$. Time-fractional NLPDEs come from classical NLPDEs by replacing its time derivative with a fractional derivative. Time-fractional partial differential equations have many applications in areas such as electrochemistry, diffusion processes, electromagnetic, material science, chaotic dynamics, and so forth [27, 29].

In this work, we consider the class of three-dimensional fourth-order time-fractional parabolic PDEs of the form

$$
\begin{array}{r}
\frac{\partial^{2 \alpha} u}{\partial t^{2 \alpha}}+\mu(x, y, z) \frac{\partial^{4} u}{\partial x^{4}}+\lambda(x, y, z) \frac{\partial^{4} u}{\partial y^{4}}+\eta(x, y, z) \frac{\partial^{4} u}{\partial z^{4}}=g(x, y, z) \\
a<x, y, z<b, t>0 .
\end{array}
$$

Subjected to the initial conditions,

$$
\begin{aligned}
u(x, y, z, 0) & =f_{0}(x, y, z), \\
\frac{\partial}{\partial t} u(x, y, z, 0) & =f_{1}(x, y, z) .
\end{aligned}
$$

And boundary conditions

$$
\begin{aligned}
u(a, y, z, t) & =g_{0}(y, z, t), \\
u(b, y, z, t) & =g_{1}(y, z, t), \\
u(x, a, z, t) & =k_{0}(x, z, t), \\
u(x, b, z, t) & =k_{1}(y, z, t), \\
u(x, y, a, t) & =k_{0}(x, y, t), \\
u(x, y, b, t) & =k_{1}(x, y, t), \\
\frac{\partial^{2} u(a, y, z, t)}{\partial x^{2}} & =\bar{g}_{0}(y, z, t), \\
\frac{\partial^{2} u(b, y, z, t)}{\partial x^{2}} & =\bar{g}_{1}(y, z, t), \\
\frac{\partial^{2} u(x, a, z, t)}{\partial y^{2}} & =\bar{k}_{0}(x, z, t), \\
\frac{\partial^{2} u(x, y, b, t)}{\partial z^{2}} & =\bar{k}_{1}(x, y, t), \\
\frac{\partial^{2} u(x, b, z, t)}{\partial y^{2}} & =\bar{k}_{1}(y, z, t), \\
\left.\frac{\partial^{2}}{2}, a, t\right) & \bar{k}_{0}(x, y, t),
\end{aligned}
$$

where $0<\alpha \leq 1, \mu(x, y, z), \lambda(x, y, z)$, and $\eta(x, y, z)$ are positive, and the functions $f_{i}, g_{i}, k_{i}, h_{i}, \bar{f}_{i}, \bar{g}_{i}, \bar{k}_{i}, \bar{h}_{i}$ for $i=0,1$.

The remaining sections of this study are organized as follows: in Section 2, we give some fundamental definitions and lemmas associated with fractional calculus. In Section 3, some basic definitions and properties related to the threedimensional fractional reduced differential transform method taking the fractional derivatives in the Caputo sense are presented, and some lemmas are proved. In Section 4, we present the formulation of the method. Section 5 is devoted to apply the method to solve three-dimensional time-fractional parabolic partial differential equation with variable coefficients. In Section 6, we present the numerical results and discussion. Tables and graphs are used to show the effectiveness, validity, and performance of the FRDTM for some values of $\alpha$. Finally, the conclusion is presented in Section 7.

\section{Fractional Calculus}

In this section, some basic definitions and lemmas associated with fractional calculus are presented. Some of these definitions are due to Riemann-Liouville and Caputo sense; for details, see $[2,24,30-33]$.

Definition 1. The gamma function. The gamma function $\Gamma(z)$ is simply a generalization of the factorial real arguments. The Gamma function can be defined as

$$
\Gamma(z)=\int_{0}^{\infty} e^{-t} t^{z-1} \mathrm{~d} t, \quad z \in \mathbb{C} .
$$

Definition 2. The fractional derivative of $f(t)$ in Caputo sense is defined as

$$
D_{t}^{\alpha} f(t)=J^{n-\alpha} D^{n} f(t)= \begin{cases}\frac{1}{\Gamma(n-\alpha)} \int_{0}^{t}(t-\tau)^{n-\alpha-1} f^{(n)}(\tau) \mathrm{d} \tau, & n-1<\alpha \leq n, \\ \frac{d^{n}}{\mathrm{~d} t^{n}} f(t), & \alpha=n .\end{cases}
$$

The basic properties of the Caputo fractional derivative can be given by the following lemma.

Lemma 1. If $m-1<\alpha \leq m, m \in \mathbb{N}$ and $f \in C_{\mu}^{m}, \mu \geq-1$, then

$$
\begin{aligned}
& D^{\alpha} J^{\alpha} f(x)=f(x), \quad x>0, \\
& D^{\alpha} J^{\alpha} f(x)=f(x)-\sum_{k=0}^{m} f^{(k)}\left(0^{+}\right) \frac{x^{k}}{k !}, \quad x>0 .
\end{aligned}
$$

Definition 3. For $p$ to be the smallest integer that exceeds $\alpha$, and $m$ to be the smallest integer that exceeds, $\beta$ the Caputo time-fractional derivative of order $\alpha>0$ is defined as 


$$
D_{t}^{\alpha} u(x, y, z, t)=\frac{\partial^{\alpha} u(x, y, z, t)}{\partial t^{\alpha}}= \begin{cases}\frac{1}{\Gamma(p-\alpha)} \int_{0}^{t}(t-\xi)^{p-\alpha-1} \frac{\partial^{p} u(x, y, z, \xi)}{\partial \xi^{p}} \mathrm{~d} \xi, & p-1<\alpha<p, \\ \frac{\partial^{\alpha}(x, y, z, t)}{\partial t^{\alpha}}, & \alpha=p \in N,\end{cases}
$$

and the space fractional derivative operator with respect to $x$, $y$, and $z$ of order $\beta>0$ is, respectively, defined as

$$
\begin{aligned}
& D_{t}^{\beta} u(x, y, z, t)=\frac{\partial^{\beta} u(x, y, z, t)}{\partial x^{\beta}}= \begin{cases}\frac{1}{\Gamma(n-\beta)} \int_{0}^{t}(t-\xi)^{n-\beta-1} \frac{\partial^{n} u(\beta, y, z, t)}{\partial \xi^{n}} \mathrm{~d} \xi, & n-1<\beta<n, \\
\frac{\partial^{\beta} u(x, y, z, t)}{\partial x^{\beta}}, & \beta=n \in N,\end{cases} \\
& D_{t}^{\beta} u(x, y, z, t)=\frac{\partial^{\beta} u(x, y, z, t)}{\partial y^{\beta}}= \begin{cases}\frac{1}{\Gamma(m-\beta)} \int_{0}^{t}(t-\xi)^{m-\beta-1} \frac{\partial^{m} u(x, \xi, z, t)}{\partial \xi^{m}} \mathrm{~d} \xi, & m-1<\beta<m, \\
\frac{\partial^{\beta} u(x, y, z, t)}{\partial y^{\beta}}, & \beta=m \in N,\end{cases} \\
& D_{t}^{\beta} u(x, y, z, t)=\frac{\partial^{\beta} u(x, y, z, t)}{\partial z^{\beta}}= \begin{cases}\frac{1}{\Gamma(m-\beta)} \int_{0}^{t}(t-\xi)^{m-\beta-1} \frac{\partial^{m} u(x, y, \xi, t)}{\partial \xi^{m}} \mathrm{~d} \xi, & m-1<\beta<m, \\
\frac{\partial^{\beta}(x, y, z, t)}{\partial z^{\beta}}, & \beta=m \in N .\end{cases}
\end{aligned}
$$

\section{Fractional Reduced Differential Transform Method (FRDTM)}

In this section, the basic definition and properties related to the three-dimensional fractional reduced differential transform method taking the fractional derivatives in the Caputo sense are presented.

Definition 4 (see $[33,34])$. If $w(x, y, z, t)$ is analytic and continuously differentiable with respect to space variables $x$, $y$, and time variable $t$ in the domain of interest, then the spectrum function is defined as

$$
\begin{aligned}
R_{D}(w(x, y, z, t)) & \approx W_{k}(x, y, z) \\
& =\frac{1}{\Gamma(k \alpha+1)}\left[\frac{\partial^{k \alpha}}{\partial t^{k \alpha}} u(x, y, z, t)\right]_{t=t_{0}} .
\end{aligned}
$$

Definition 5 (see $[33,34]$ ). The inverse FRDTM of $W_{k}(x, y, z)$ is defined as

$$
\begin{aligned}
R_{D}^{-1}\left[W_{k}(x, y, z)\right] & \approx w(x, y, z, t) \\
& =\sum_{k=0}^{\infty} \frac{1}{\Gamma(k \alpha+1)} W_{k}(x, y, z)\left(t-t_{o}\right)^{k \alpha}, \\
w(x, y, z, t) & =\sum_{k=0}^{\infty} \frac{1}{\Gamma(k \alpha+1)}\left[\frac{\partial^{k}}{\partial t^{k}} w(x, y, z, t)\right]_{t=t_{0}} .
\end{aligned}
$$

The inverse transform of the set of values of $\left[U_{k}(x, y, z)\right]_{k=0}^{n}$ gives the $n$-terms approximate solution as follows:

$$
\bar{u}_{n}(x, y, z, t)=\sum_{k=0}^{n} U_{k}(x, y, z) t^{\alpha k} .
$$

Therefore, the exact solution of the problem is given by

$$
u(x, y, z, t)=\lim _{n \longrightarrow \infty} \bar{u}_{n}(x, y, z, t)=\sum_{k=0}^{\infty} U_{k}(x, y, z) t^{\alpha k}
$$


Lemma 2. If $w(x, y, z, t)=f(x, y, z) u(x, y, z, t)$, then $W_{k}(x, y, z)=f(x, y, z) U_{k}(x, y, z)$.

Proof. By Definition 3 and properties of FRDTM given in Table 1, we have

$$
\begin{aligned}
& W_{k}(x, y, z)=\frac{1}{\Gamma(k \alpha+1)}\left[\frac{\partial^{k \alpha}}{\partial t^{k \alpha}} w(x, y, z, t)\right]_{t=t_{0}} \\
& W_{k}(x, y, z)=\frac{1}{\Gamma(k \alpha+1)}\left[\frac{\partial^{k \alpha}}{\partial t^{k \alpha}} f(x, y, z) u(x, y, z, t)\right]_{t=t_{0}} \\
& W_{k}(x, y, z)=f(x, y, z) \frac{1}{\Gamma(k \alpha+1)}\left[\frac{\partial^{k \alpha}}{\partial t^{k \alpha}} u(x, y, z, t)\right]_{t=t_{0}} \\
& W_{k}(x, y, z)=f(x, y, z) U_{k}(x, y, z)
\end{aligned}
$$

Lemma 3. If $w(x, y, z, t)=f(x, y, z) \sin (\alpha x+\beta y+\mu z+$ $\omega t)$, then $W_{k}(x, y, z)=f(x, y, z)\left(\omega^{k} / k !\right) \sin (\alpha x+\beta y+\mu z+$ $(\pi k / 2)) . \alpha, \beta, \mu$, and $\omega$ are the constants.

Proof. By Definition 3 and properties of FRDTM given in Table 1, we have

$$
\begin{aligned}
& W_{k}(x, y, z)=\frac{1}{\Gamma(k \alpha+1)}\left[\frac{\partial^{k \alpha}}{\partial t^{k \alpha}} w(x, y, z, t)\right]_{t=t_{0}}, \\
& W_{k}(x, y, z)=\frac{1}{\Gamma(k \alpha+1)}\left[\frac{\partial^{k \alpha}}{\partial t^{k \alpha}} f(x, y, z) \sin (\alpha x+\beta y+\mu z+\omega t)\right]_{t=t_{0}}, \\
& W_{k}(x, y, z)=f(x, y, z) \frac{1}{\Gamma(k \alpha+1)}\left[\frac{\partial^{k \alpha}}{\partial t^{k \alpha}} \sin (\alpha x+\beta y+\mu z+\omega t)\right]_{t=t_{0}},
\end{aligned}
$$

$W_{k}(x, y, z)=f(x, y, z)\left(\omega^{k} / k !\right) \sin (\alpha x+\beta y+\mu z+(\pi k / 2))$. $\alpha, \beta, \mu$, and $\omega$ are the constants.

Lemma 4. If $w(x, y, z, t)=f(x, y, z) \cos (\alpha x+\beta y+\mu z+$ $\omega t)$, then $W_{k}(x, y, z)=f(x, y, z)\left(\omega^{k} / k !\right) \cos (\alpha x+\beta y+\mu z+$ $(\pi k / 2)) . \alpha, \beta, \mu$, and $\omega$ are the constants.

The proof is similar to Lemma 3.

If $\alpha=\beta=\mu=0$, then Lemma 4 reduces to $W_{k}(x$, $y, z)=f(x, y, z)\left(\omega^{k} / k !\right) \cos (\pi k / 2)$, where $k=0,1,2, \ldots$

\section{Solution of the Problem by FRDTM}

The steps to find the general solution of the governing equation (1) subjected to initial conditions (2) by the proposed method are as follows:

Applying the properties of FRDTM given in Table 1 to equations (1) and (2), we obtain

$$
\begin{gathered}
\frac{\Gamma(\alpha(k+2)+1)}{\Gamma(k \alpha+1)} U_{k+2}(x, y, z)+\mu(x, y, z) \frac{\partial^{4} U_{k}(x, y, z)}{\partial x^{4}} \\
+\lambda(x, y, z) \frac{\partial^{4} U_{k}(x, y, z)}{\partial y^{4}} \\
+\eta(x, y, z) \frac{\partial^{4} U_{k}(x, y, z)}{\partial z^{4}}=G_{k}(x, y, z), \\
U_{0}(x, y, z)=f_{0}(x, y, z), \\
U_{1}(x, y, z)=f_{1}(x, y, z) .
\end{gathered}
$$

Solving for $U_{k+2}(x, y, z)$ from equation (15), we get

$$
U_{k+2}(x, y, z)=\frac{-\Gamma(k \alpha+1)}{\Gamma(\alpha(k+2)+1)}\left[\begin{array}{c}
\mu(x, y, z) \frac{\partial^{2} U_{k}(x, y, z)}{\partial x^{2}}+\lambda(x, y, z) \frac{\partial^{2} U_{k}(x, y, z)}{\partial y^{2}}+ \\
\eta(x, y, z) \frac{\partial^{4} U_{k}(x, y, z)}{\partial z^{4}}-G_{k}(x, y, z)
\end{array}\right]
$$

When $k=0,1,2,3, \ldots$ by iterative calculations, we obtain the following successive terms:

$$
\begin{aligned}
& U_{2}(x, y, z)=\frac{-1}{\Gamma(2 \alpha+1)}\left[\mu(x, y, z) \frac{\partial^{4} f_{0}(x, y, z)}{\partial x^{4}}+\lambda(x, y, z) \frac{\partial^{4} f_{0}(x, y, z)}{\partial y^{4}}+\eta(x, y, z) \frac{\partial^{4} f_{0}(x, y, z)}{\partial z^{4}}-G_{0}(x, y, z)\right] \\
& U_{3}(x, y, z)=\frac{-\Gamma(\alpha+1)}{\Gamma(3 \alpha+1)}\left[\mu(x, y, z) \frac{\partial^{2} f_{1}(x, y, z)}{\partial x^{2}}+\lambda(x, y, z) \frac{\partial^{2} f_{1}(x, y, z)}{\partial y^{2}}+\eta(x, y, z) \frac{\partial^{4} f_{1}(x, y, z)}{\partial z^{4}}-G_{1}(x, y, z)\right],
\end{aligned}
$$


TABLE 1: The basic properties of FRDTM are described below $[33,34]$.

\begin{tabular}{lc}
\hline The original function & Transformed form \\
\hline$w(x, y, z, t)=\alpha u(x, y, z, t) \pm \beta v(x, y, z, t)$ & $W_{k}(x, y, z)=\alpha U_{k}(x, y, z)+\beta V_{k}(x, y, z)$ \\
$w(x, y, z, t)=\left(\partial^{N \alpha} / \partial t^{N \alpha}\right) u(x, y, z, t)$ & $W_{k}(x, y, z)=(\Gamma[\alpha(k+N)+1] / \Gamma[k \alpha+1]) U_{k+N}(x, y, z)$ \\
$w(x, y, z, t)=f(x, y, z) u(x, y, z, t)$ & $W_{k}(x, y, z)=f(x, y, z) U_{k}(x, y, z)$ \\
$w(x, y, z, t)=\sin (\alpha x+\beta y+\mu z+\omega t)$ & $W_{k}(x, y, z)=\left(\omega^{k} / k !\right) \sin (\alpha x+\beta y+\mu z+(\pi k / 2)), \alpha, \beta, \mu$, and $\omega$ are the constants \\
$w(x, y, z, t)=\cos (\alpha x+\beta y+\mu z+\omega t)$ & $W_{k}(x, y, z)=\left(\omega^{k} / k !\right) \cos (\alpha x+\beta y+\mu z+(\pi k / 2)), \alpha, \beta, \mu$, and $\omega$ are the constants \\
\hline
\end{tabular}

and so on.

Thus, the inverse transform of the set of values of $\left[U_{k}(x, y, z)\right]_{k=0}^{n}$ giving the $n$-terms approximate solution $u_{n}(x, y, z, t)=\sum_{k=0}^{n} U_{k}(x, y, z) t^{\alpha k}$ and the FRDTM solution of equation (1) subjected to the initial condition (2) is given by

$$
\begin{aligned}
u(x, y, z, t)= & \lim _{n \longrightarrow \infty} u_{n}(x, y, z, t)=\sum_{k=0}^{\infty} U_{k}(x, y, z) t^{\alpha k} \\
= & f_{o}(x, y, z)+f_{1}(x, y, z) t^{\alpha}+\frac{-1}{\Gamma(2 \alpha+1)} \\
& \cdot\left[\mu(x, y, z) \frac{\partial^{4} f_{o}(x, y, z)}{\partial x^{4}}+\lambda(x, y, z) \frac{\partial^{4} f_{o}(x, y, z)}{\partial y^{4}} \eta(x, y, z) \frac{\partial^{4} f_{o}(x, y, z)}{\partial z^{2}}-G_{o}(x, y, z)\right] t^{2 \alpha}+\cdots
\end{aligned}
$$

\section{Illustrative Examples}

Example 1. Consider the following partial differential equation in three space variables

$$
\begin{aligned}
& \frac{\partial^{2 \alpha} u}{\partial t^{2 \alpha}}+\left(\frac{y+z}{2 \cos x}-1\right) \frac{\partial^{4} u}{\partial x^{4}}+\left(\frac{z+x}{2 \cos y}-1\right) \frac{\partial^{4} u}{\partial y^{4}} \\
& \quad+\left(\frac{x+y}{2 \cos z}-1\right) \frac{\partial^{4} u}{\partial z^{4}}=0, \quad 0<x, y, z<\frac{\pi}{3}, 0<\alpha \leq 1, t>0,
\end{aligned}
$$

subjected to the initial conditions

$$
\begin{array}{r}
u(x, y, z, 0)=(x+y+z)-(\cos x+\cos y+\cos z), \\
\frac{\partial u}{\partial t}(x, y, z, 0)=\cos x+\cos y+\cos z-(x+y+z) .
\end{array}
$$

When $\alpha=1$, equation (20) reduces to the classical (or nonfractional) three-dimensional homogeneous parabolic partial differential equation [35-37].

Applying the properties of FRDTM given in Table 1 and Lemma 2 to equations (20) and (21), we get

$$
\begin{aligned}
U_{k+2} & =\frac{-\Gamma(k \alpha+1)}{\Gamma(\alpha(k+2)+1)}\left[\left(\frac{y+z}{2 \cos x}-1\right) \frac{\partial^{4} U_{k}}{\partial x^{4}}+\left(\frac{z+x}{2 \cos y}-1\right) \frac{\partial^{4} U_{k}}{\partial y^{4}}+\left(\frac{x+y}{2 \cos z}-1\right) \frac{\partial^{4} U_{k}}{\partial z^{4}}\right], \\
U_{0}(x, y, z) & =x+y+z-(\cos x+\cos y+\cos z), \\
U_{1}(x, y, z) & =\cos x+\cos y+\cos z-(x+y+z) .
\end{aligned}
$$

When $k=0,1,2, \ldots$, we have 
$U_{2}(x, y, z)=\frac{1}{\Gamma(2 \alpha+1)}[x+y+z-(\cos x+\cos y+\cos z)]$

$U_{3}(x, y, z)=\frac{-\Gamma(\alpha+1)}{\Gamma(3 \alpha+1)}[x+y+z-(\cos x+\cos y+\cos z)]$,

$U_{4}(x, y, z)=\frac{1}{\Gamma(4 \alpha+1)}[x+y+z-(\cos x+\cos y+\cos z)]$,

$U_{5}(x, y, z)=\frac{-\Gamma(\alpha+1)}{\Gamma(5 \alpha+1)}[x+y+z-(\cos x+\cos y+\cos z)]$,

and so on. Continuing in this way, the remaining steps of the FRDTM can be obtained. Then, by equation (19), we obtain the following approximate solution:

$$
\begin{aligned}
u(x, y, z, t)= & {[(x+y+z)-(\cos x+\cos y+\cos z)] } \\
& \cdot\left[1-t^{\alpha}+\frac{1}{\Gamma(2 \alpha+1)} t^{2 \alpha}-\frac{\Gamma(\alpha+1)}{\Gamma(3 \alpha+1)} t^{3 \alpha}\right. \\
& \left.+\frac{1}{\Gamma(4 \alpha+1)} t^{4 \alpha}-\cdots\right] .
\end{aligned}
$$

The exact solution of the classical form of Example 1 is $u(x, y, z, t)=(x+y+z-\cos x-\cos y-\cos z) e^{-t}$ as in [35-37].
Example 2. Consider the following three-dimensional nonhomogeneous fourth-order parabolic partial differential equation:

$$
\begin{aligned}
& \frac{\partial^{2 \alpha} u}{\partial t^{2 \alpha}}+\left(\frac{1}{4 ! z}\right) \frac{\partial^{4} u}{\partial x^{4}}+\left(\frac{1}{4 ! x}\right) \frac{\partial^{4} u}{\partial y^{4}}+\left(\frac{1}{4 ! y}\right) \frac{\partial^{4} u}{\partial z^{4}} \\
& =\left[-\frac{x}{y}-\frac{y}{z}-\frac{z}{x}+\frac{1}{x^{5}}+\frac{1}{y^{5}}+\frac{1}{z^{5}}\right] \cos t, \quad \frac{1}{2}<x, y, z<1,
\end{aligned}
$$

$0<\alpha \leq 1, t>0$

subjected to initial conditions

$$
\begin{aligned}
u(x, y, z, 0) & =\frac{x}{y}+\frac{y}{z}+\frac{z}{x}, \\
\frac{\partial}{\partial t} u(x . y, z, 0) & =0 .
\end{aligned}
$$

When $\alpha=1$, equation (25) reduces to the classical (or nonfractional) three-dimensional nonhomogeneous parabolic partial differential equation [37].

Applying properties of FRDTM given in Table 1 and Lemma 4 to equations (25) and (26), we get

$$
\begin{aligned}
\frac{\Gamma(\alpha(k+2)+1)}{\Gamma(\alpha k+1)} U_{k+2}(x, y, z)+\left(\frac{1}{4 ! z}\right) \frac{\partial^{4} U_{k}(x, y, z)}{\partial x^{4}}+\left(\frac{1}{4 ! x}\right) \frac{\partial^{4} U_{k}(x, y, z)}{\partial y^{4}}+ \\
\left(\frac{1}{4 ! y}\right) \frac{\partial^{4} U_{k}(x, y, z)}{\partial z^{4}}=\left[-\frac{x}{y}-\frac{y}{z}-\frac{z}{x}+\frac{1}{x}+\frac{1}{y}+\frac{1}{z}\right] \frac{1}{k !} \cos \frac{\pi}{2} k, \\
U_{0}(x, y, z)=\frac{x}{y}+\frac{y}{z}+\frac{z}{x}, \\
U_{1}(x . y, z)=0 .
\end{aligned}
$$

Solving for $U_{k+2}(x, y, z)$ from equation (27), we have

$$
U_{k+2}(x, y, z)=\frac{-\Gamma(\alpha k+1)}{\Gamma(\alpha(k+2)+t 1)}\left[\begin{array}{c}
\left(\frac{1}{4 ! z}\right) \frac{\partial^{4} U_{k}(x, y, z)}{\partial x^{4}}+\left(\frac{1}{4 ! x}\right) \frac{\partial^{4} U_{k}(x, y, z)}{\partial y^{4}}+\left(\frac{1}{4 ! y}\right) \frac{\partial^{4} U_{k}(x, y, z)}{\partial z^{4}}- \\
{\left[-\frac{x}{y}-\frac{y}{z}-\frac{z}{x}+\frac{1}{x^{5}}+\frac{1}{y^{5}}+\frac{1}{z^{5}}\right] \frac{1}{k !} \cos \frac{\pi}{2} k}
\end{array}\right] .
$$

Now, when $k=0,1,3, \ldots$, 


$$
\begin{aligned}
& U_{2}(x, y, z)=\frac{-1}{\Gamma(2 \alpha+1)}\left[\frac{x}{y}+\frac{y}{z}+\frac{z}{x}\right], \\
& U_{3}(x, y, z)=0, \\
& U_{4}(x, y, z)=\frac{-\Gamma(2 \alpha+1)}{\Gamma(4 \alpha+1)}\left[\frac{-1}{\Gamma(2 \alpha+1)}\left(\frac{1}{x^{5}}+\frac{1}{y^{5}}+\frac{1}{z^{5}}\right)+\left[-\frac{x}{y}-\frac{y}{z}-\frac{z}{x}+\frac{1}{x^{5}}+\frac{1}{y^{5}}+\frac{1}{z^{5}}\right] \frac{1}{2 !}\right], \\
& U_{5}(x, y, z)=0,
\end{aligned}
$$

$$
U_{6}(x, y, z)=\frac{-\Gamma(4 \alpha+1)}{\Gamma(6 \alpha+1)}\left[\begin{array}{c}
\left(\frac{1}{\Gamma(4 \alpha+1)}\right)\left(\frac{70}{x^{9} z}\right)+\frac{-\Gamma(2 \alpha+1)}{2 \Gamma(4 \alpha+1)}\left(\frac{-1}{x^{5}}+\frac{70}{x^{9} z}\right)+ \\
\left(\frac{1}{\Gamma(4 \alpha+1)}\right)\left(\frac{70}{y^{9} x}\right)+\left(\frac{-\Gamma(2 \alpha+1)}{2 \Gamma(4 \alpha+1)}\right)\left(\frac{-1}{y^{5}}+\frac{70}{y^{9} x}\right)+ \\
\left(\frac{1}{\Gamma(4 \alpha+1)}\right)\left(\frac{70}{z^{9} y}\right)+\left(\frac{-\Gamma(2 \alpha+1)}{2 \Gamma(4 \alpha+1)}\right)\left(\frac{-1}{z^{5}}+\frac{70}{z^{9} y}\right)- \\
{\left[-\frac{x}{y}-\frac{y}{z}-\frac{z}{x}+\frac{1}{x^{5}}+\frac{1}{y^{5}}+\frac{1}{z^{5}}\right] \frac{1}{4 !}}
\end{array}\right],
$$

and so on. Continuing in this way, the remaining steps of the FRDTM can be obtained. Then, by equation (19), we obtain

$$
\begin{aligned}
u(x, y, z, t)= & \left(\frac{x}{y}+\frac{y}{z}+\frac{z}{x}\right)-\left(\frac{x}{y}+\frac{y}{z}+\frac{z}{x}\right) \frac{1}{\Gamma(2 \alpha+1)} t^{2 \alpha}+\frac{-\Gamma(2 \alpha+1)}{\Gamma(4 \alpha+1)} \\
& \cdot\left[\frac{-1}{\Gamma(2 \alpha+1)}\left(\frac{1}{x^{5}}+\frac{1}{y^{5}}+\frac{1}{z^{5}}\right)+\left[-\frac{x}{y}-\frac{y}{z}-\frac{z}{x}+\frac{1}{x^{5}}+\frac{1}{y^{5}}+\frac{1}{z^{5}}\right] \frac{1}{2 !}\right] t^{4 \alpha}+\cdots
\end{aligned}
$$

The exact solution of the classical form of Example 2 is $u(x, y, z, t)=((x / y)+(y / z)+(z / x))\left(1-(1 / 2 !) t^{2}+(1 / 4 !)\right.$ $\left.t^{4}-\cdots\right)=((x / y)+(y / z)+(z / x)) \cos t$ as in [37].

\section{Numerical Results and Discussion}

In this section, the numerical values of the function $u(x, y, z$, $t$ ) of Examples 1 and 2 are computed for various values of the fractional order $\alpha$, and the exact solutions for the variables $x$, $y, z$, and $t$ are presented in tables and figures.

Tables 2 and 3 exhibit the behavior of FRDTM solutions for different values of fractional order $\alpha$ and the absolute errors $(E)$ approximated in the $5^{\text {th }}$ and $6^{\text {th }}$ order at different values of the independent variables $x, y, z$, and time $t$ of equations (20) and (25), respectively. As it can be seen from these tables, when the value of the fractional order $\alpha$ becomes close to 1 , the obtained FRDTM solutions of Examples 1 and 2 are close to the exact solutions of its respective classical (nonfractional) equations obtained by different methods. In particular, when $\alpha=1$, the FRDTM solutions of the mentioned examples are in excellent agreement with the exact solutions of their corresponding classical (nonfractional) form, for details, see [35-37].

Figures 1(a)-1(d) show the evaluation results of the approximate analytical solution of equation (20). These figures also show the behavior of the approximate solution obtained by the proposed method at fixed values $x=0.6$ and $y=0.7$ and for different values of the fractional order $\alpha=0.5$, 0.7, 1 and the exact solution.

Figures 2(a) and 2(b) demonstrate the solutions $u(x, y, z, t)$ verses $t$ of equation (20) for a fixed value of $x, y$, and $z$. In Figure 2(a), the FRDTM solutions at $\alpha=0.7,0.8,1$ and the exact solution are compared for a fixed value $x=y=z=0.4$. In Figure 2(b), the FRDTM solutions at $\alpha=0.7,0.8,1$ and the exact solution are compared for a fixed value $x=y=z=0.6$.

Figures 3(a)-3(d) show the evaluation results of the approximate analytical solution of equation (25). These figures also depict the behavior of the approximate solutions obtained by the proposed method at fixed values $x=0.6$ and $y=0.7$ and for a different time-fractional order $\alpha=0.5,0.7,1$ and the exact solution. 
TABLE 2: Fifth-order approximate numerical solution of equation (20) at different values of the fractional order $\alpha$ and the absolute error $E=\mid$ $u_{\text {exact }}-u_{\alpha=1} \mid$ for $u(x, y, z, t)$ by FRDTM.

\begin{tabular}{|c|c|c|c|c|c|c|c|c|c|}
\hline \multirow[b]{2}{*}{$x$} & \multirow[b]{2}{*}{$y$} & & & \multicolumn{4}{|c|}{ FRDTM for different values of $\alpha$} & \multirow[b]{2}{*}{$u_{\text {exact }}$} & \multirow{2}{*}{ Error $(E)$} \\
\hline & & $z$ & $t$ & $\alpha=0.5$ & $\alpha=0.7$ & $\alpha=0.9$ & $\alpha=1$ & & \\
\hline \multirow{4}{*}{0.1} & \multirow{4}{*}{0.3} & \multirow{2}{*}{0.2} & 0.3 & -1.57206 & -1.61233 & -1.68227 & -1.72641 & -1.72641 & 0 \\
\hline & & & 0.5 & -1.47993 & -1.43721 & -1.41053 & -1.41342 & -1.41346 & $4 E-05$ \\
\hline & & \multirow{2}{*}{0.8} & 0.3 & -0.97616 & -1.00116 & -1.04459 & -1.072 & -1.072 & 0 \\
\hline & & & 0.5 & -0.918948 & -0.892426 & -0.875856 & -0.877649 & -0.877679 & $3 E-05$ \\
\hline \multirow{4}{*}{0.4} & \multirow{4}{*}{0.6} & \multirow{2}{*}{0.2} & 0.3 & -1.02973 & -1.05611 & -1.10192 & -1.13083 & -1.13083 & 0 \\
\hline & & & 0.5 & -0.969381 & -0.941403 & -0.923924 & -0.925816 & -0.925847 & $3.1 E-05$ \\
\hline & & \multirow[b]{2}{*}{0.8} & 0.3 & -0.43383 & -0.444942 & -0.464243 & -0.476422 & -0.476423 & $1 E-06$ \\
\hline & & & 0.5 & -0.408403 & -0.396616 & -0.389252 & -0.390049 & -0.390062 & $1.3 E-05$ \\
\hline \multirow{4}{*}{0.7} & \multirow{4}{*}{0.9} & \multirow{2}{*}{0.2} & 0.3 & -0.382167 & -0.391956 & -0.408958 & -0.419687 & -0.419687 & 0 \\
\hline & & & 0.5 & -0.359768 & -0.349384 & -0.342897 & -0.3436 & -0.343611 & $1.1 E-05$ \\
\hline & & \multirow{2}{*}{0.8} & 0.3 & 0.213737 & 0.219212 & 0.228721 & 0.234721 & 0.234722 & $1 E-06$ \\
\hline & & & 0.5 & 0.20121 & 0.195403 & 0.191775 & 0.192167 & 0.192174 & $7 E-06$ \\
\hline \multirow{4}{*}{1} & \multirow{4}{*}{1} & \multirow{2}{*}{0.2} & 0.3 & 0.0939895 & 0.096397 & 0.100579 & 0.103217 & 0.103217 & 0 \\
\hline & & & 0.5 & 0.0884808 & 0.0859271 & 0.0843317 & 0.0845044 & 0.0845072 & $2.8 E-06$ \\
\hline & & \multirow{2}{*}{0.8} & 0.3 & 0.689893 & 0.707565 & 0.738258 & 0.757625 & 0.757626 & $1 E-06$ \\
\hline & & & 0.5 & 0.649459 & 0.630714 & 0.619004 & 0.620271 & 0.620292 & $2.1 E-05$ \\
\hline
\end{tabular}

TABLE 3: Sixth-order approximate numerical solution of equation (25) at different values of the fractional order $\alpha$ and the absolute error $E=\left|u_{\text {exact }}-u_{\alpha=1}\right|$ for $u(x, y, z, t)$ by FRDTM.

\begin{tabular}{|c|c|c|c|c|c|c|c|c|c|}
\hline \multirow{2}{*}{$x$} & \multirow{2}{*}{$y$} & $\tau$ & $t$ & \multicolumn{4}{|c|}{ FRDTM for different values of $\alpha$} & \multirow{2}{*}{$u_{\text {exact }}$} & \multirow{2}{*}{ Error $(E)$} \\
\hline & & $z$ & $\tau$ & $\alpha=0.5$ & $\alpha=0.7$ & $\alpha=0.9$ & $\alpha=1$ & & \\
\hline \multirow{4}{*}{0.6} & \multirow{4}{*}{0.65} & \multirow{2}{*}{0.6} & 0.5 & -287.047 & -15.1344 & 2.09635 & 2.63837 & 2.63837 & 0 \\
\hline & & & 1 & -2317.74 & -321.166 & -16.9664 & 1.6243 & 1.62437 & $7 E-05$ \\
\hline & & \multirow{2}{*}{0.8} & 0.5 & 2.33312 & 2.50559 & 2.6387 & 2.69322 & 2.69322 & 0 \\
\hline & & & 1 & 1.65806 & 1.65806 & 1.65806 & 1.65806 & 1.65814 & $8 E-05$ \\
\hline \multirow{4}{*}{0.7} & \multirow{4}{*}{0.75} & \multirow{2}{*}{0.6} & 0.5 & 2.3115 & 2.48237 & 2.61425 & 2.66827 & 2.66827 & 0 \\
\hline & & & 1 & 1.6427 & 1.6427 & 1.6427 & 1.6427 & 1.64278 & $8 E-05$ \\
\hline & & \multirow{2}{*}{0.8} & 0.5 & 2.29114 & 2.46051 & 2.59122 & 2.64476 & 2.64476 & 0 \\
\hline & & & 1 & 1.62823 & 1.62823 & 1.62823 & 1.62823 & 1.6283 & $7 E-05$ \\
\hline \multirow{4}{*}{0.8} & \multirow{4}{*}{0.85} & \multirow{2}{*}{0.6} & 0.5 & 2.36272 & 2.53738 & 2.67217 & 2.72739 & 2.72739 & 0 \\
\hline & & & 1 & 1.6791 & 1.6791 & 1.6791 & 1.6791 & 1.67917 & $7 E-05$ \\
\hline & & \multirow{2}{*}{0.8} & 0.5 & 2.28352 & 2.45233 & 2.58261 & 2.63597 & 2.63597 & 0 \\
\hline & & & 1 & 1.62282 & 1.62282 & 1.62282 & 1.62282 & 1.62289 & $7 E-05$ \\
\hline \multirow{4}{*}{0.9} & \multirow{4}{*}{0.9} & \multirow{2}{*}{0.6} & 0.5 & 2.40744 & 2.5854 & 2.72275 & 2.77901 & 2.77901 & 0 \\
\hline & & & 1 & 1.71088 & 1.71088 & 1.71088 & 1.71088 & 1.71096 & $8 E-05$ \\
\hline & & \multirow{2}{*}{0.8} & 0.5 & 2.29129 & 2.46067 & 2.59139 & 2.64494 & 2.64494 & 0 \\
\hline & & & 1 & 1.62834 & 1.62834 & 1.62834 & 1.62834 & 1.62841 & $7 E-05$ \\
\hline
\end{tabular}

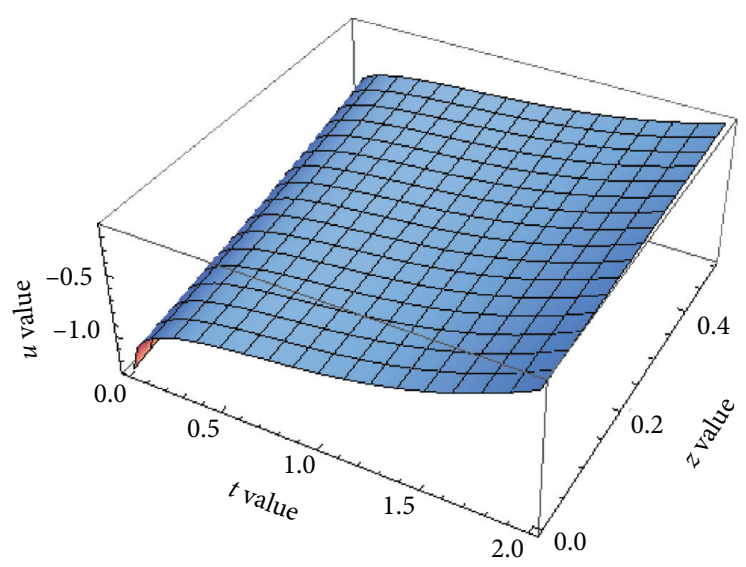

(a)

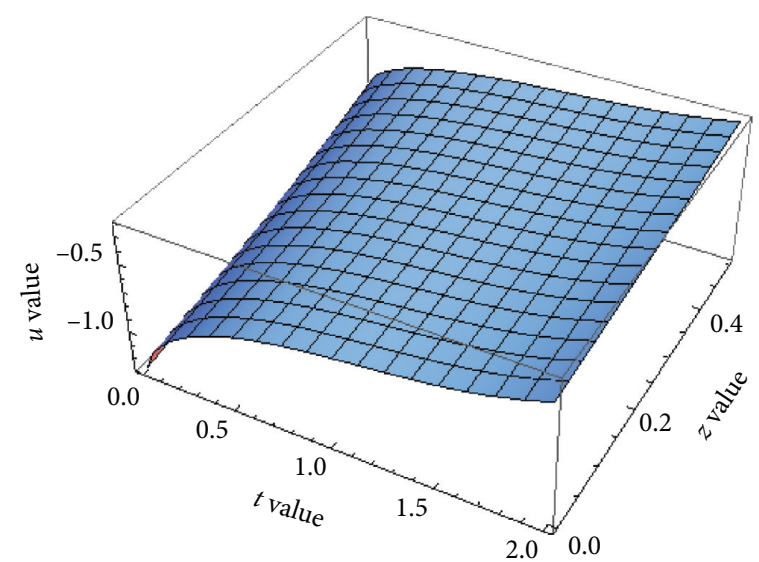

(b)

FIgURe 1: Continued. 


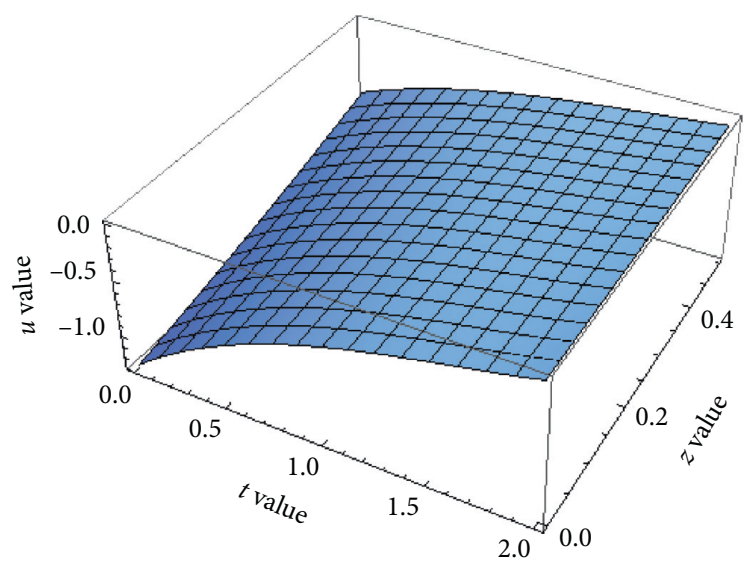

(c)

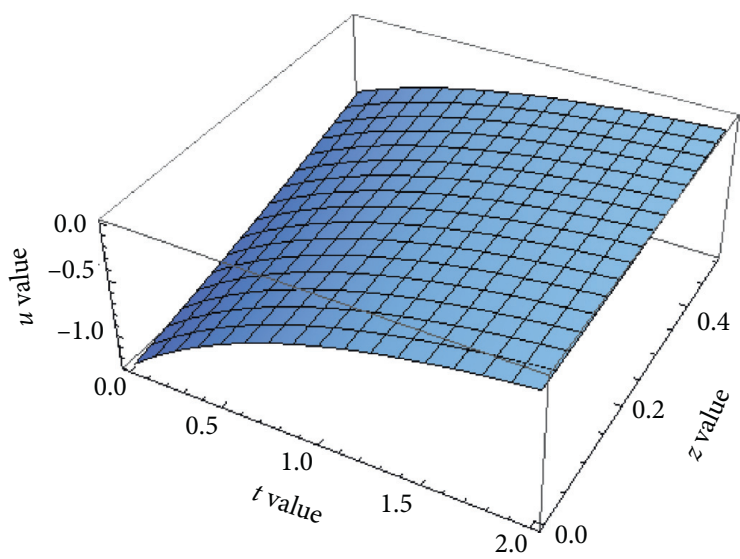

(d)

FIgURE 1: 3D plots of FRDTM solutions of equation (20) for the different fractional order $\alpha$ and the exact solution.

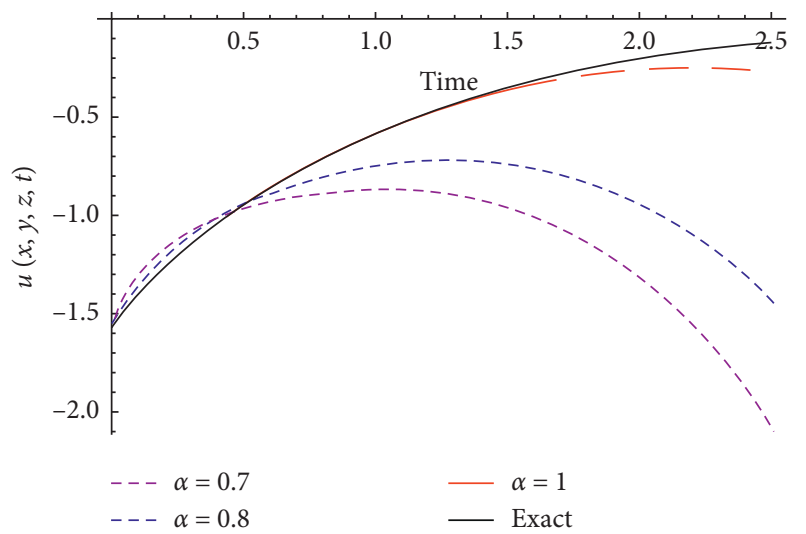

(a)

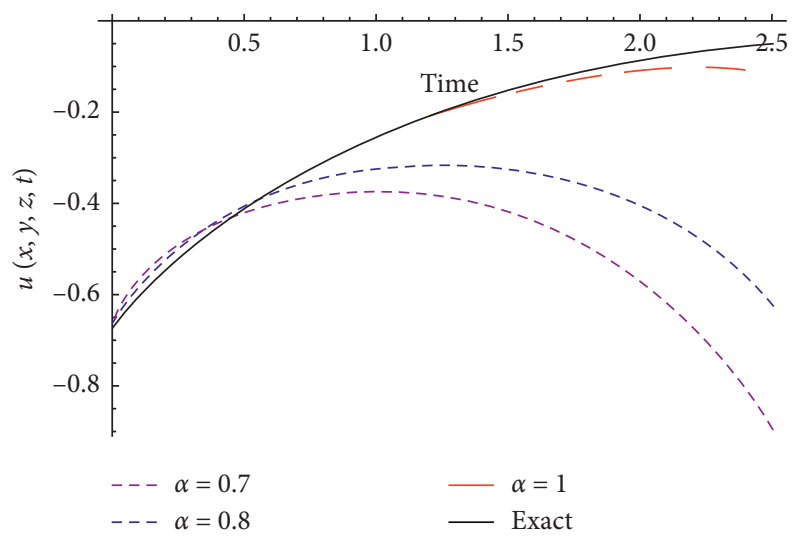

(b)

FIgURE 2: 2D plots of FRDTM solutions of equation (20) for the different fractional order $\alpha$ and the exact solution.

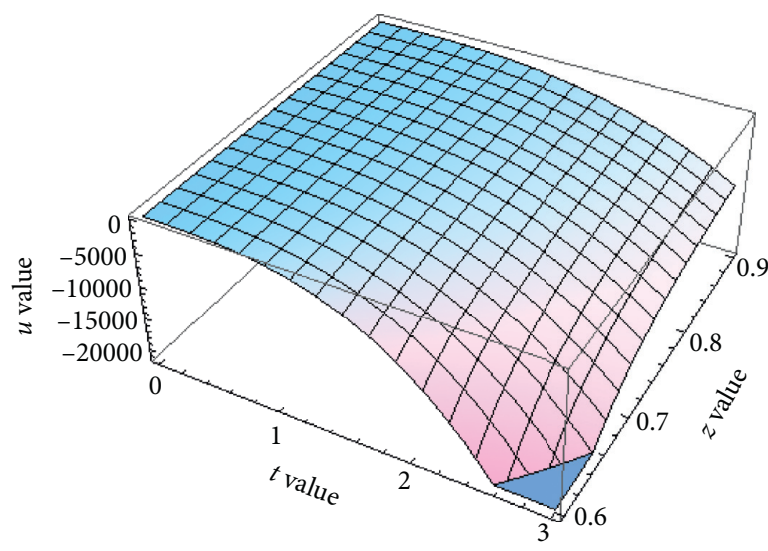

(a)

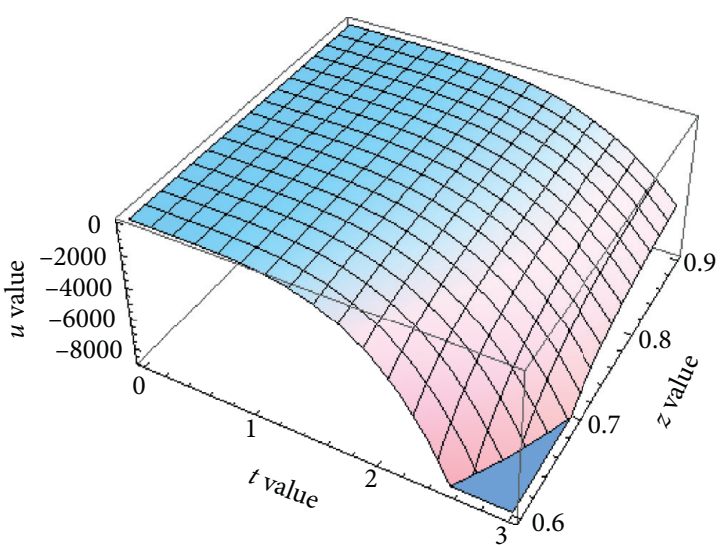

(b)

FIgURE 3: Continued. 


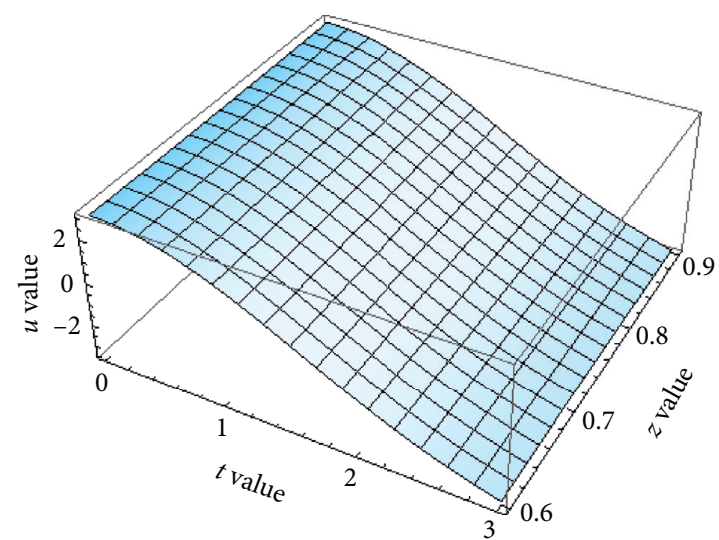

(c)

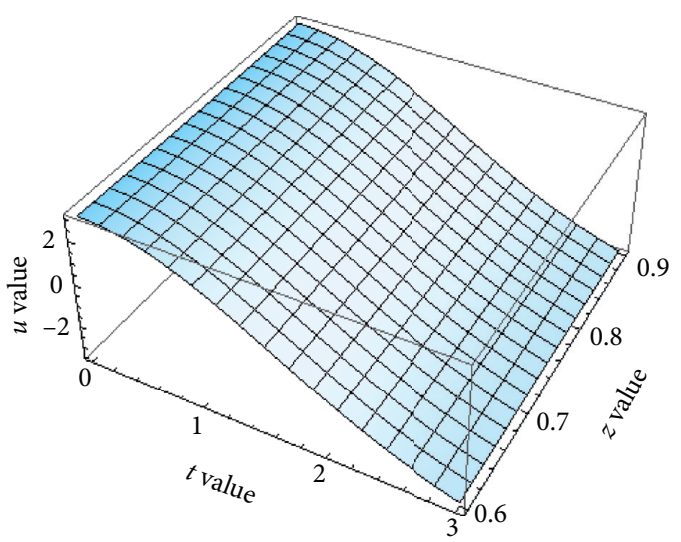

(d)

FIGURE 3: 3D plots of FRDTM solutions of equation (25) for the different fractional order $\alpha$ and the exact solution.

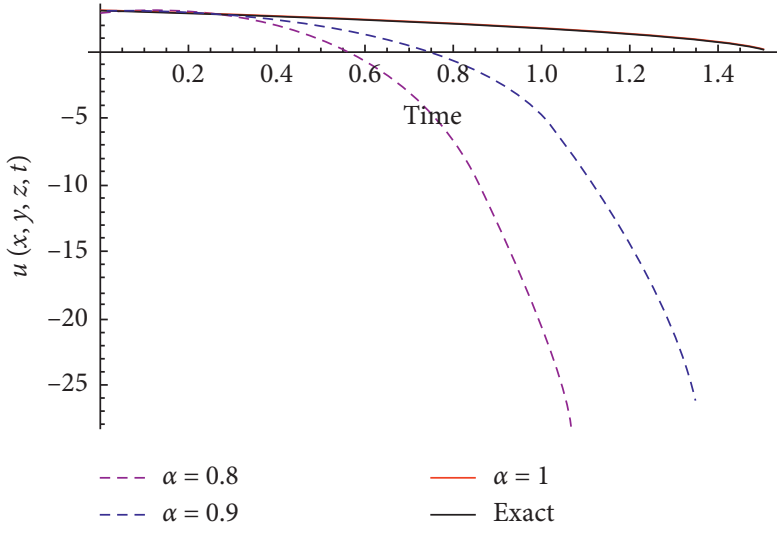

(a)

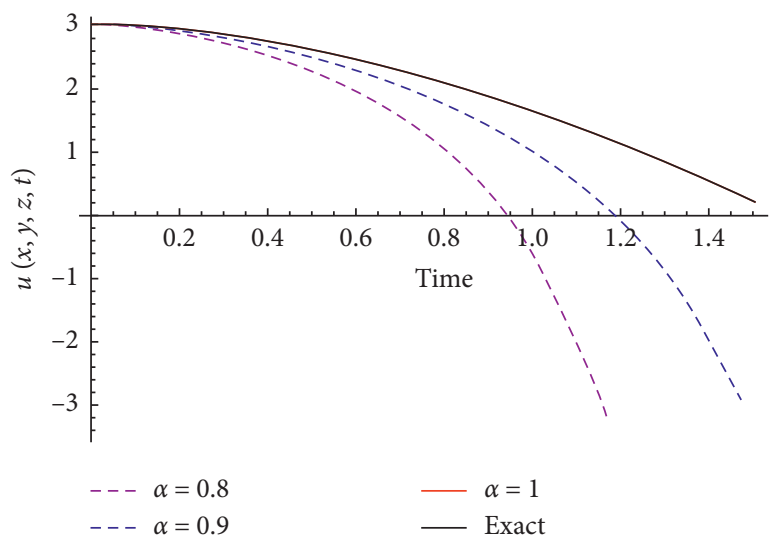

(b)

FIGURE 4: 2D plots of FRDTM solutions of (25) for the different fractional order $\alpha$ and the exact solution.

Figures 4(a) and 4(b) demonstrate the solutions $u(x, y, z, t)$ verses $t$ of equation (25) for a fixed value of $x, y, z$. In Figure 4(a), the FRDTM solutions at $\alpha=0.8,0.9,1$ and exact solution are compared for a fixed value $x=y=z=0.8$. In Figure 4(b), the FRDTM solutions at $\alpha=$ $0.8,0.9,1$ and exact solution are compared for a fixed value $x=y=z=0.9$.

\section{Conclusion}

In this study, we successfully employed the FRDTM to find the approximate and exact solutions of three-dimensional fourth-order time-fractional parabolic partial differential equations with variable coefficients taking the fractional derivative in Caputo sense. It is found that the results we obtained in Examples 1 and 2 when $\alpha=1$ are in excellent agreement with the exact solutions obtained by VIM [35], ADM [36], and RDTM [37]. Also, the illustrated examples demonstrate that the suggested technique can be observed as a fast, simple, and efficient tool for computing three-dimensional fourth-order time-fractional parabolic partial differential equations (PDEs). The main advantage of the method is that it can be applied to fractional PDE's and obtain approximate and exact solutions of fractional PDEs without requiring any discretization, perturbation, or restrictive conditions. Moreover, the tables and graphs constructed in this work demonstrate the physical behavior of the FRDTM solutions of the problems for different values of $\alpha$. As a result, we conclude that the FRDTM is very powerful, straightforward, and effective to obtain approximate and exact solutions of a wide variety problems related to fractional PDEs applied in sciences such as mathematics, physics, and engineering.

\section{Data Availability}

The data used to support the findings of this study are included within the article.

\section{Conflicts of Interest}

The authors declare that there are no conflicts of interest. 


\section{Authors' Contributions}

YOM and Ademe KG proposed the main idea of this study. YOM, Ademe KG, and Ayana DN equally contributed in making the first draft and revision and approved the final manuscript for submission.

\section{Acknowledgments}

The authors would like to thank Jimma University, College of Natural Sciences, and Department of Mathematics for providing the necessary resources during conducting this study.

\section{References}

[1] Y. Chatibi, E. H. El Kinani, and A. Ouhadan, "Variational calculus involving nonlocal fractional derivative with MittagLeffler kernel," Chaos, Solitons \& Fractals, vol. 118, pp. 117-121, 2019.

[2] I. Podlubny, Fractional Differential Equations, Academic Press, San Diego, CA, USA, 1999.

[3] B. A. Tayyan and A. H. Sakka, "Lie symmetry analysis of some conformable fractional partial differential equations," Arabian Journal of Mathematics, vol. 9, pp. 201-212, 2020.

[4] Y. Chatibi, E. H. El Kinani, and A. Ouhadan, "Lie symmetry analysis of conformable differential equations," AIMS Mathematics, vol. 4, no. 4, pp. 1133-1144, 2019.

[5] M. Bishehniasar, S. Salahshour, A. Ahmadian, F. Ismail, and D. Baleanu, "An Accurate Approximate- Analytical Technique for Solving Time-Fractional Partial Differential equations," Complexity, vol. 2017, Article ID 8718209, 12 pages, 2017.

[6] D. Baleanu, J. A. T. Machado, and A. C. J. Luo, Fractional Dynamics and Control, Springer Science \& Business Media, New York, NY, USA, 2011.

[7] S. Das, "Analytical solution of a fractional diffusion equation by variational iteration method," Computers \& Mathematics with Applications, vol. 57, no. 3, pp. 483-487, 2009.

[8] A. Elsaid, "The variational iteration method for solving Riesz fractional partial differential equations," Computers \& Mathematics with Applications, vol. 60, no. 7, pp. 1940-1947, 2010.

[9] Y. Chatibi, E. H. El Kinani, and A. Ouhadan, "On the discrete symmetry analysis of some classical andfractional differential equations," Mathematical Methods in the Applied Sciences, pp. 1-11, 2019.

[10] Y. Chatibi, E. H. El Kinani, and A. Ouhadan, "Lie symmetry analysis and conservation laws for the time fractional BlackScholes equation," International Journal of Geometric Methods in Modern Physics, vol. 17, no. 1, Article ID 2050010, 2020.

[11] J. H. He, "Homotopy perturbation method: a new nonlinear analytical technique," Applied Mathematics and Computation, vol. 135, pp. 73-79, 2003.

[12] F. Tchier, M. Inc, and A. Yusuf, "Symmetry analysis, exact solutions and numerical approximations for the space-time Carleman equation in nonlinear dynamical systems," European Physical Journal Plus, vol. 134, pp. 1-18, 2019.

[13] A. Yusuf, M. Inc, and M. Bayram, "Soliton solutions for Kudryashov-Sinelshchikov equation," Sigma Journal of Engineering and Natural Sciences, vol. 37, pp. 439-444, 2019.
[14] M. Inc, A. Yusuf, A. I. Aliyu et al., "Dark and singular optical Solitons for the conformable space time nonlinear Schrodinger equation with Kerr and power law nonlinearity," Optik, vol. 162, pp. 65-75, 2018.

[15] M. Inc, A. Yusuf, A. I. Aliyu et al., "Lie symmetry analysis and explicit solutions for the time generalized Burgers-Huxley equation," Optical and Quantum Electronics, vol. 50, pp. 1-16, 2018.

[16] A. Akgul, "Reproducing kernel method for fractional derivative with non-local and non-singular kernel," in Fractional Derivatives with Mittag-Leffler Kernel, Series of Studies in Systems, Decision and Control Springer, Cham, Switzerland, 2019.

[17] A. Akgül, "A novel method for a fractional derivative with non-local and non-singular kernel," Chaos, Solitons \& Fractals, vol. 114, pp. 478-482, 2018.

[18] M. G. Sakar, O. Saldir, and A. Akgul, "A novel technique for fractional Bagley-Torvik equation," IN Proceedings of the National Academy of Sciences, India, Section A: Physical Sciences, vol. 89, no. 2, pp. 1-7, 2018.

[19] M. Modanli and A. Akgül, "Numerical solution of fractional telegraph differential equations by theta-method," The European Physical Journal Special Topics, vol. 226, no. 16-18, pp. 3693-3703, 2017.

[20] E. K. Akgul, "Solutions of the linear and nonlinear differential equations within the generalized fractional derivatives," Chaos, vol. 29, no. 2, Article ID 023108, 2019.

[21] E. K. Akgül, B. Orcan, and A. Akgül, "Solving higher-order fractional differential equations by reproducing kernel Hilbert space method," Journal of Advanced Physics, vol. 7, no. 1, pp. 98-102, 2018.

[22] Y. Keskin and G. Oturanc, "Reduced differential transform method: a new approach to fractional partial differential equations," Nonlinear Science Letters A, vol. 1, pp. 61-72, 2010.

[23] V. K. Srivastava, M. K. Awasthi, and M. Tamsir, "RDTM solution of Caputo time fractional-order hyperbolic Telegraph equation," AIP Advances, vol. 3, Article ID 032142, 2013.

[24] V. K. Srivastava, M. K. Awasthi, and S. Kumar, "Analytical approximations of two and three dimensional time-fractional telegraphic equation by reduced differential transform method," Egyptian Journal of Basic and Applied Sciences, vol. 1, no. 1, pp. 60-66, 2014.

[25] V. K. Srivastava, S. Kumar, M. K. Awasthi, and B. K. Singh, "Two-dimensional time fractional-order biological population model and its analytical solution," Egyptian Journal of Basic and Applied Sciences, vol. 1, no. 1, pp. 71-76, 2014.

[26] V. K. Srivastava, N. Mishra, S. Kumar, B. K. Singh, and M. K. Awasthi, "Reduced differential transform method for solving $(1+\mathrm{n})$-dimensional Burgers' equation," Egyptian Journal of Basic and Applied Sciences, vol. 1, no. 2, pp. 115119, 2014.

[27] D.M. Yadeta, A. K. Gizaw, and Y. O. Mussa, "Approximate analytical solution of one-dimensional Beam equations by using time-fractional reduced differential transform method," Journal of Applied Mathematics, vol. 2020, Article ID 7627385 , 13 pages, 2020.

[28] M. Amin, M. Abbas, M. K. Iqbal, and D. Baleanu, "Nonpolynomial quintic spline for numerical solution of fourthorder time fractional partial differential equations," Advances in Difference Equations, vol. 183, 2019.

[29] H. Tariq and G. Akram, "Quintic spline technique for time fractional fourth-order partial differential equation," $\mathrm{Nu}$ merical Methods for Partial Differential Equations, vol. 33, no. 2, pp. 445-466, 2017. 
[30] B. K. Singh, "Fractional reduced differential transform method for numerical computation of a system of linear and nonlinear fractional partial differential equations," International Journal of Open Problems in Computer Science and Mathematics, vol. 9, no. 3, pp. 20-38, 2016.

[31] M. S. Rawashdeh, "A reliable method for the space-time fractional Burgers and time-fractional CahnAllen equations via the FRDTM," Advances in Difference Equations, vol. 99, p. 14, 2017.

[32] S. Abuasad, I. Hashim, and S. A. Abdul Karim, "Modified fractional reduced differential transform method for the solution of multiterm time-fractional diffusion equations," Advances in Mathematical Physics, vol. 2019, Article ID 5703916, 14 pages, 2019.

[33] D. Lu, J. Wang, M. Arshad, Abdullah, and A. Ali, "Fractional reduced differential transform method for space-time fractional order Heat-like and wave-like partial differential equations," Journal of Advanced Physics, vol. 6, no. 4, pp. 598-607, 2018.

[34] M. Arshad, D. Lu, and J. Wang, " $(N+1)$-dimensional fractional reduced differential transform method for fractional order partial differential equations," Communications in Nonlinear Science and Numerical Simulation, vol. 48, pp. 509-519, 2017.

[35] J. Biazar and H. Ghazvini, "He's variational iteration method for fourth-order parabolic equations," Computers \& Mathematics with Applications, vol. 54, no. 7-8, pp. 1047-1054, 2007.

[36] A.-M. Wazwaz, "Exact solutions for variable coefficients fourth-order parabolic partial differential equations in higherdimensional spaces," Applied Mathematics and Computation, vol. 130, no. 2-3, pp. 415-424, 2002.

[37] B. Ibis, "Application of reduced differential transformation method for solving fourth-order parabolic partial differential equations," Journal of Mathematics and Computer Science, vol. 12, no. 02 , pp. 124-131, 2014. 